Pacific Journal of Mathematics

EXTENSION OF THE
HARDY-LITTLEWOOD-FEFFERMAN-STEIN INEQUALITY 


\section{EXTENSION OF THE \\ HARDY-LITTLEWOOD-FEFFERMAN-STEIN \\ INEQUALITY}

\section{AKIHITO UCHIYAMA}

We will show inequalities concerning the functions of the form $f * t^{-n} \varphi(\cdot / t)(x)$ defined on $R_{+}^{n+1}$ and give their applications to real Hardy spaces. These inequalities can be regarded as weak extensions of the Hardy-Littlewood-Fefferman-Stein inequality concerning harmonic functions.

1. Introduction. In C. Fefferman and E. M. Stein [6] (p. 172 Lemma 2), (see also Hardy and Littlewood [8]), they showed

THEOREM 1.A. Let $u(x)$ be a complex-valued harmonic function defined on

$$
B=\left\{x=\left(x_{1}, \ldots, x_{n}\right) \in R^{n}: \sum_{j=1}^{n} x_{j}^{2}<1\right\} .
$$

Let $p>0$. Then

$$
|u(0)|^{p} \leq C \int_{B}|u(x)|^{p} d x
$$

where $C$ is a constant depending only on $p$ and $n$.

Consequently, if $u(x, t)$ is harmonic on $R_{+}^{n+1}=\left\{(x, t): x \in R^{n}\right.$, $t>0\}$ and if $p>0$, then we have

$$
|u(0,1)|^{p} \leq C \int_{|x|<1} d x \int_{1 / 2}^{3 / 2}|u(x, t)|^{p} d t .
$$

This inequality has some interesting applications to the theory of real Hardy spaces. (See [6].)

In this paper we show analogous inequalities for functions of the form $f * t^{-n} \varphi(\cdot / t)(x)$ defined on $R_{+}^{n+1}$, where $f \in \bigcup_{1 \leq p \leq+\infty} L^{p}\left(R^{n}\right)$ is arbitrary and where $\varphi \in C\left(R^{n}\right) \cap \bigcap_{1 \leq p \leq+\infty} L^{p}\left(R^{n}\right)$ satisfies certain conditions. Our results have weaker forms than (1.1) but still they have some interesting applications to real Hardy spaces.

First we prepare several definitions. 
Functions considered are complex-valued and measurable. Sets considered are measurable. $D_{t}$ denotes $\partial / \partial t$. For a multi-index $\gamma=\left(\gamma_{1}, \ldots, \gamma_{n}\right)$, where $\gamma_{1}, \ldots, \gamma_{n}$ are nonnegative integers, $D_{x}^{\gamma}$ and $l(\gamma)$ denote $\partial^{\gamma_{1}+\cdots+\gamma_{n}} / \partial x_{1}^{\gamma_{1}} \cdots \partial x_{n}^{\gamma_{n}}$ and $\gamma_{1}+\cdots+\gamma_{n}$, respectively. For $f \in L^{2}\left(R^{n}\right)$, $\mathscr{F} f$ denotes its Fourier transform. For $x=\left(x_{1}, \ldots, x_{n}\right) \in R^{n},|x|$ denotes $\left(\sum_{j=1}^{n} x_{j}^{2}\right)^{1 / 2}$. For $(x, t) \in R_{+}^{n+1}, B(x, t)$ denotes $\left\{y \in R^{n}:|x-y|<t\right\}$. $(\varphi)_{t}(x)$ denotes $t^{-n} \varphi(x / t)$. For a real number $\alpha,[\alpha]$ denotes its integral part.

For $\kappa \in L_{\mathrm{loc}}^{1}\left(R^{n}\right)$ and $\alpha>0$ let

$$
\|\kappa\|_{\Lambda_{\alpha}}=\sup _{B} \inf _{P: \operatorname{deg} P \leq \alpha}|B|^{-1-\alpha / n} \int_{B}|\kappa(x)-P(x)| d x,
$$

where the supremum is taken over all balls $B$ in $R^{n},|B|$ denotes the Lebesgue measure of $B$ and where the infimum is taken over all polynomials $P(x)$ of degree $\leq \alpha$. Let

Let

$$
\Lambda_{\alpha}\left(R^{n}\right)=\left\{\kappa \in L_{\mathrm{loc}}^{1}\left(R^{n}\right):\|\kappa\|_{\Lambda_{\alpha}}<+\infty\right\} .
$$

$$
\begin{aligned}
& \mathscr{B}_{\alpha}\left(R^{n}\right)=\left\{\kappa \in \Lambda_{\alpha}\left(R^{n}\right): \operatorname{supp} \kappa \subset B(0,1),\|\kappa\|_{\Lambda_{\alpha}} \leq 1\right\}, \\
& \mathscr{B}_{\alpha}^{\prime}\left(R^{n}\right)=\left\{\kappa \in \Lambda_{\alpha}\left(R^{n}\right)\right. \text { : there exists a sequence of functions } \\
& \left\{\kappa_{j}\right\}_{j=0}^{\infty} \subset \mathscr{B}_{\alpha}\left(R^{n}\right) \text { such that } \\
& \left.\kappa(x)=\sum_{j=0}^{\infty} 2^{-j}\left(\kappa_{j}\right)_{2^{j}}(x)\right\}, \\
& \mathscr{B}_{\alpha}^{0}\left(R^{n}\right)=\left\{\kappa \in \mathscr{B}_{\alpha}\left(R^{n}\right): \int_{R^{n}} \kappa(x) d x=0\right\}, \\
& \mathscr{B}_{\alpha}^{0 \prime}\left(R^{n}\right)=\left\{\kappa \in \Lambda_{\alpha}\left(R^{n}\right):\right. \text { there exists a sequence of functions } \\
& \left.\left\{\kappa_{j}\right\}_{j=0}^{\infty} \subset \mathscr{B}_{\alpha}^{0}\left(R^{n}\right) \text { such that (1.2) holds }\right\} \text {. }
\end{aligned}
$$

REMARK 1.1. Notice the following simple fact: if $0<\beta<\alpha$ and if $\varphi \in \mathscr{B}_{\alpha}\left(R^{n}\right)$ (or $\varphi \in \mathscr{B}_{\alpha}^{\prime}\left(R^{n}\right)$ ), then $c \varphi \in \mathscr{B}_{\beta}\left(R^{n}\right)$ (or $c \varphi \in \mathscr{B}_{\beta}^{\prime}\left(R^{n}\right)$ ), where $c$ is a positive constant depending only on $\alpha, \beta$ and $n$.

Definition 1.1. For $\alpha>0$ and $\varphi \in \Lambda_{\alpha}\left(R^{n}\right)$ we say that $\varphi$ satisfies the condition (I. $\alpha$ ) if the following three conditions hold:

(I. $\alpha .1) \quad \varphi \in \mathscr{B}_{\alpha}^{\prime}\left(R^{n}\right)$,

(I. $\alpha$.2) $\mathscr{F} \varphi(\xi)$ is $(n+[\alpha]+2)$-times differentiable except $\xi=0$ and

$$
\left|D_{\xi}^{\gamma \mathscr{F}} \varphi(\xi)\right| \leq|\xi|^{1-l(\gamma)}, \quad \xi \neq 0,
$$

for any $\gamma$ with $1 \leq 1(\gamma) \leq \mathrm{n}+[\alpha]+2$,

(I. $\alpha .3) \quad \mathscr{F} \varphi(0) \neq 0$. 
Definition 1.2. For $\alpha>0$ and $\left\{\varphi_{1}, \varphi_{2}, \ldots, \varphi_{N}\right\} \subset \Lambda_{\alpha}\left(R^{n}\right)$ we say that the set of functions $\left\{\varphi_{1}, \varphi_{2}, \ldots, \varphi_{N}\right\}$ satisfies the condition (II. $\alpha$ ) if the following three conditions hold:

(II. $\alpha .1) \quad \varphi_{i} \in \mathscr{B}_{\alpha}^{0 \prime}\left(R^{n}\right), i=1,2, \ldots, N$,

(II. $\alpha .2) \quad \mathscr{F} \varphi_{i}(\xi)(i=1,2, \ldots, N)$ are $(n+[\alpha]+2)$-times differentiable except $\xi=0$ and

$$
\left|D_{\xi}^{\gamma} \mathscr{F} \varphi_{i}(\xi)\right| \leq|\xi|^{1-l(\gamma)}, \quad \xi \neq 0, i=1,2, \ldots, N,
$$

for any $\gamma$ with $0 \leq l(\gamma) \leq n+[\alpha]+2$,

(II. $\alpha .3) \quad \inf _{\xi \in R^{n} \backslash\{0\}} \sup _{t>0} \sum_{i=1}^{N}\left|\mathscr{F} \varphi_{i}(t \xi)\right|>0$.

For the sake of simplicity we put

$$
\delta=\text { the left-hand side of (II. } \alpha .3 \text { ). }
$$

EXAMPLE 1.1. Let

$$
P(x, t)=C_{n} t\left(|x|^{2}+t^{2}\right)^{-(n+1) / 2}
$$

that is the Poisson kernel. Then $c P(x, 1)$ satisfies the condition (I. $\alpha)$ for any $\alpha>0$ if $c(>0)$ is small enough depending on $\alpha$ and $n$.

EXAmple 1.2. Let $\varphi \in \mathscr{S}\left(R^{n}\right)$ and $\int_{R^{n}} \varphi(x) d x \neq 0$. Then $c \varphi$ satisfies the condition (I. $\alpha)$ for any $\alpha>0$ if $c(>0)$ is small enough depending only on $\alpha$ and $\varphi$.

EXAmple 1.3. Let $N=1$ and $\varphi(x)=c D_{t} P(x, 1)$. Then $\{\varphi\}$ satisfies the condition (II. $\alpha$ ) for any $\alpha>0$ if $c(>0$ ) is small enough depending on $\alpha$ and $n$.

EXAMPLE 1.4. Let $N=n$ and

$$
\varphi_{i}(x)=c D_{x_{i}} P(x, 1), \quad i=1,2, \ldots, n .
$$

Then the set of functions $\left\{\varphi_{1}, \varphi_{2}, \ldots, \varphi_{n}\right\}$ satisfies the condition (II. $\alpha$ ) for any $\alpha>0$ if $c(>0)$ is small enough depending on $\alpha$ and $n$.

EXAMPLE 1.5. Let $\varphi_{1}, \varphi_{2}, \ldots, \varphi_{N} \in \mathscr{S}\left(R^{n}\right)$,

$$
\mathscr{F} \varphi_{i}(0)=0, \quad i=1,2, \ldots, N,
$$

and let (II. $\alpha .3)$ hold. Then the set of functions $\left\{c \varphi_{1}, \ldots, c \varphi_{N}\right\}$ satisfies the 
condition (II. $\alpha$ ) for any $\alpha>0$ if $c(>0)$ is small enough depending on $\alpha$ and $\left\{\varphi_{1}, \ldots, \varphi_{N}\right\}$.

REMARK 1.2. The author learned the condition (II. $\alpha .3)$ from A. Calderón and Torchinsky [1], in which they investigated the area integrals defined from the kernels that satisfy the conditions of Example 1.5.

Our first result is the following.

\section{THEOREM 1.1. Let}

$$
0<\beta<\alpha, \quad 0<\varepsilon<1 \text { and } 0<q \leq 1 .
$$

Let $\varphi \in \Lambda_{\alpha}\left(R^{n}\right)$ satisfy the condition (I. $\left.\alpha\right)$. Let $\kappa \in \mathscr{B}_{\beta}^{\prime}\left(R^{n}\right)$ and $f \in$ $\bigcup_{1 \leq p \leq+\infty} L^{p}\left(R^{n}\right)$. Then

$$
\begin{aligned}
& \left|\int_{R^{n}} f(x) \kappa(x) d x\right| \\
& \quad \leq C\left(\iint_{R_{+}^{n+1}}\left|f *(\varphi)_{t}(y)\right|^{q} k_{\beta, \varepsilon}(y, t)^{q} t^{n(q-1)-1} d y d t\right)^{1 / q},
\end{aligned}
$$

where

$$
k_{\beta, \varepsilon}(y, t)=t^{\beta}(1+|y|+t)^{-n-\beta-1+\varepsilon}
$$

and where $C$ is a constant depending only on $\alpha, \beta, \varepsilon, q, \mathscr{F} \varphi(0)$ and $n$.

If $\varphi, \alpha$ and $\beta$ are as in Theorem 1.1, then it follows from Remark 1.1 that $c \varphi \in \mathscr{B}_{\beta}^{\prime}\left(R^{n}\right)$ provided $c(>0)$ is small enough. Thus substituting $\kappa(x)=\varphi(-x)$ into (1.5) we get

$$
|f * \varphi(0)| \leq C\left(\iint_{R_{+}^{n+1}}\left|f *(\varphi)_{t}(y)\right|^{q} k_{\beta, \varepsilon}(y, t)^{q} t^{n(q-1)-1} d y d t\right)^{1 / q} .
$$

Applying this inequality to the case of Example 1.1, i.e. $(\varphi)_{t}(x)=P(x, t)$, and putting

$$
u(x, t)=(f * P(\cdot, t))(x)
$$

we get

$$
\begin{aligned}
|u(0,1)|^{p} & \leq C\left(\iint_{R_{+}^{n+1}}|u(y, t)|^{q} k_{\beta, \varepsilon}(y, t)^{q} t^{n(q-1)-1} d y d t\right)^{p / q} \\
& \leq C \iint_{R_{+}^{n+1}}|u(y, t)|^{p} k_{\beta, \varepsilon}(y, t)^{q} t^{n(q-1)-1} d y d t
\end{aligned}
$$


for any $\beta>0$, any $q \in(n /(n+\beta), 1]$, any $p \in[q,+\infty)$ and any $\varepsilon \in$ $(0,1)$, where $C$ depends only on $\beta, q, p, \varepsilon$ and $n$. (The second inequality follows from Hölder's inequality and from the fact that

$$
\iint k_{\beta, \varepsilon}(y, t)^{q} t^{n(q-1)-1} d y d t<+\infty
$$

provided $q>n /(n+\beta)$.) This is the reason why we regard Theorem 1.1 as a weak extension of the inequality (1.1).

If we replace the condition (I. $\alpha$ ) by the condition (II. $\alpha$ ), then we have the following.

THEOREM 1.2. Let (1.4) hold. Let $\left\{\varphi_{1}, \varphi_{2}, \ldots, \varphi_{N}\right\} \subset \Lambda_{\alpha}\left(R^{n}\right)$ satisfy the condition (II. $\alpha$ ). Let $\kappa \in \mathscr{B}_{\beta}^{0 \prime}\left(R^{n}\right)$ and $f \in \bigcup_{1 \leq p \leq+\infty} L^{p}\left(R^{n}\right)$. Then

$$
\begin{aligned}
& \left|\int_{R^{n}} f(x) \kappa(x) d x\right| \\
& \quad \leq C \sum_{i=1}^{N}\left(\iint_{R_{+}^{n+1}}\left|f *\left(\varphi_{i}\right)_{t}(y)\right|^{q} k_{\beta, \varepsilon}(y, t)^{q} t^{n(q-1)-1} d y d t\right)^{1 / q},
\end{aligned}
$$

where $k_{\beta, \varepsilon}$ is defined by (1.6) and where $C$ is a constant depending only on $\alpha, \delta, \beta, \varepsilon, q, N$ and $n$. (For the definition of $\delta$ recall (1.3).)

We prove Theorems 1.1 and 1.2 in $\$ \S 4-6$. In $\S \S 2$ and 3 we give applications of these theorems to real Hardy spaces. In $\$ 2$ using Theorem 1.1 we give another proof of the estimate of grand maximal functions in terms of radial maximal functions. In $\$ 3$ using Theorems 1.1 and 1.2 and using the ideas suggested by Robert Fefferman we give another proof of the estimate of the Lusin $S$-functions in terms of the Littlewood-Paley $g$-functions. Since we do not need harmonicity at all in Theorems 1.1 and 1.2 , we can develop these arguments in general setting.

Notation. For $q>0, f \in L_{\mathrm{loc}}^{1}\left(R^{n}\right)$ and $x \in R^{n}$ let

$$
M_{q}(f)(x)=\sup _{B: B \ni x}\left(|B|^{-1} \int_{B}|f(y)|^{q} d y\right)^{1 / q},
$$

where the supremum is taken over all balls $B\left(\subset R^{n}\right)$ that contain $x . \check{f}(x)$ denotes $f(-x)$. For a measurable set $E, \chi_{E}(x)$ denotes the characteristic function of $E$. For $(x, s) \in R_{+}^{n+1}$ let

$$
\begin{aligned}
Q(x, s) & =\left\{(y, t) \in R_{+}^{n+1}: 0<t \leq s,|x-y| \leq s\right\} \\
Q^{\prime}(x, s) & =\left\{(y, t) \in R_{+}^{n+1}: s / 2 \leq t \leq 2 s,|x-y| \leq s / 2\right\}
\end{aligned}
$$


and

$$
Q^{\prime \prime}(x, s)=\left\{(y, t) \in R_{+}^{n+1}: 3 s / 4 \leq t \leq 3 s / 2,|x-y| \leq s / 6\right\} .
$$

The letter $C$ denotes various positive constants.

Acknowledgment. The author would like to thank Professor Robert Fefferman at the University of Chicago for very inspiring conversations to whom the author owes the fundamental ideas in $\S 3$.

2. Grand maximal functions from radial maximal functions. In this section we give an application of Theorem 1.1. Let $\alpha>0$ and let $\varphi$ satisfy the condition (I. $\alpha)$. We fix $\varphi$. For $f \in \cup_{1 \leq p \leq+\infty} L^{p}\left(R^{n}\right)$ we define a radial maximal function $f_{\varphi}^{+}(x)$ by

$$
f_{\varphi}^{+}(x)=\sup _{t>0}\left|f *(\varphi)_{t}(x)\right| .
$$

For $f \in \bigcup_{1 \leq p \leq+\infty} L^{p}\left(R^{n}\right)$ and $\beta>0$ we define a grand maximal function $f_{\beta}^{*}(x)$ by

$$
f_{\beta}^{*}(x)=\sup \left\{\left|f *(\kappa)_{t}(x)\right|: t>0, \kappa \in \mathscr{B}_{\beta}\left(R^{n}\right)\right\} .
$$

As an application of Theorem 1.1 we get

TheOrem 2.1. Let $0<\beta<\alpha$. Let $\varphi$ satisfy the condition (I. $\alpha)$. Let $q>n /(n+\beta), x \in R^{n}$, and $f \in \bigcup_{1 \leq p \leq+\infty} L^{p}\left(R^{n}\right)$. Then

$$
f_{\beta}^{*}(x) \leq C M_{q}\left(f_{\varphi}^{+}\right)(x),
$$

where $C$ is a constant depending only on $\alpha, \beta, q, \mathscr{F} \varphi(0)$ and $n$.

Proof. We may assume $x=0$. Let $\kappa \in \mathscr{B}_{\beta}\left(R^{n}\right)$ and $\varepsilon \in(0,1)$. By Theorem 1.1 we get

$$
\begin{aligned}
|f * \kappa(0)| & \leq C\left(\iint_{R_{+}^{n+1}} f_{\varphi}^{+}(y)^{q} k_{\beta, \varepsilon}(y, t)^{q} t^{n(q-1)-1} d y d t\right)^{1 / q} \\
& =C\left(\int_{R^{n}} f_{\varphi}^{+}(y)^{q} d y \int_{0}^{+\infty} k_{\beta, \varepsilon}(y, t)^{q} t^{n(q-1)-1} d t\right)^{1 / q} \\
& \leq C\left(\int f_{\varphi}^{+}(y)^{q}(1+|y|)^{-n-q(1-\varepsilon)} d y\right)^{1 / q} \text { by } q>\frac{n}{n+\beta} \\
& \leq C M_{q}\left(f_{\varphi}^{+}\right)(0) .
\end{aligned}
$$

By the argument of dilation we get

$$
\left|f *(\kappa)_{t}(0)\right| \leq C M_{q}\left(f_{\varphi}^{+}\right)(0)
$$

for any $t>0$. Since $\kappa \in \mathscr{B}_{\beta}\left(R^{n}\right)$ is arbitrary, we have

$$
f_{\beta}^{*}(0) \leq C M_{q}\left(f_{\varphi}^{+}\right)(0) \text {. }
$$


As a consequence of Theorem 2.1, using the Hardy-Littlewood maximal theorem we get the following which was originally proved by $\mathrm{C}$. Fefferman and E. Stein [6].

Corollary 2.A. Let $\alpha, \beta$ and $\varphi$ be as in Theorem 1.1. Let $p>$ $n /(n+\beta)$ and $f \in \bigcup_{1 \leq p \leq+\infty} L^{p}\left(R^{n}\right)$. Then

$$
\left\|f_{\beta}^{*}\right\|_{L^{p}} \leq C\left\|f_{\varphi}^{+}\right\|_{L^{p}}
$$

where $C$ is a constant depending only on $\alpha, \beta, p, \mathscr{F} \varphi(0)$ and $n$.

REMARK 2.1. Theorem 2.1 had been proved by Uchiyama [13] in a somewhat different method. (See also [12].) Theorem 2.1 for the case $\varphi$ is the Poisson kernel had been proved by C. Fefferman and E. M. Stein [6] p. 170 by using the inequality (1.1). The above proof of Theorem 2.1 is a generalization of their argument.

3. $S$-functions from $g$-functions. The author owes the fundamental idea in this section to Robert Fefferman.

For $a>0, r \in R, x \in R^{n}$ and for a continuous function $u(y, t)$ defined on $R_{+}^{n+1}$ let

$$
S_{a, r}(u)(x)^{2}=a^{-n} \iint_{\Gamma(x, a)}|u(y, t)|^{2} t^{2 r-n-1} d y d t
$$

and

$$
S_{0, r}(u)(x)^{2}=\int_{0}^{+\infty}|u(x, t)|^{2} t^{2 r-1} d t
$$

where

$$
\Gamma(x, a)=\left\{(y, t) \in R_{+}^{n+1}:|x-y|<a t\right\} .
$$

Note that if $u$ is harmonic, then $S_{0,1}\left(D_{t} u\right)(x)$ and $S_{0,1}\left(D_{x_{i}} u\right)(x)$ are the Littlewood-Paley $g$-functions and $S_{1,1}\left(D_{t} u\right)(x)$ and $S_{1,1}\left(D_{x_{t}} u\right)(x)$ are the Lusin $S$-functions. Our concern is to get the estimates of $S_{a, r}(u)$ in terms of $S_{0, r}(u)$.

First we explain the idea suggested by R. Fefferman, which is also implicit in R. Fefferman and Stein [7], for the case when $u$ is harmonic. The following is crucial.

THEOREM 3.A. Let $u(y, t)$ be a complex-valued harmonic function defined on $R_{+}^{n+1}$. Let $r \in R, q>0, a \geq 0$ and $x \in R^{n}$. Then

$$
S_{a, r}(u)(x)^{2} \leq C \int_{0}^{+\infty} M_{q}(u(\cdot, t))(x)^{2} t^{2 r-1} d t,
$$

where $C$ is a constant depending only on $r, q, a$ and $n$. 
Proof. We give a proof only for the case $a=1$. We may assume $q \leq 2$. Applying the inequality (1.1) with dilation we get that if $(y, t) \in$ $\Gamma(x, 1)$, then

$$
\begin{aligned}
|u(y, t)|^{q} & \leq C t^{-n-1} \int_{B(y, t)} d z \int_{t / 2}^{3 t / 2}|u(z, s)|^{q} d s \\
& \leq C t^{-n-1} \int_{B(x, 2 t)} d z \int_{t / 2}^{3 t / 2}|u(z, s)|^{q} d s \\
& \leq C \int_{t / 2}^{3 t / 2} M_{q}(u(\cdot, s))(x)^{q} \frac{d s}{s} .
\end{aligned}
$$

Thus

$$
\begin{aligned}
& \iint_{\Gamma(x, 1)}|u(y, t)|^{2} t^{2 r-n-1} d y d t \\
& \quad \leq \int_{0}^{+\infty} t^{2 r-1} d t\left(C \int_{t / 2}^{3 t / 2} M_{q}(u(\cdot, s))(x)^{q} \frac{d s}{s}\right)^{2 / q} \\
& \quad=\int_{0}^{+\infty} t^{2 r-1} d t\left(C \int_{1 / 2}^{3 / 2} M_{q}(u(\cdot, s t))(x)^{q} \frac{d s}{s}\right)^{2 / q} \\
& \quad \leq C \int_{0}^{+\infty} M_{q}(u(\cdot, t))(x)^{2} t^{2 r-1} d t
\end{aligned}
$$

by $2 / q \geq 1$ and by Minkowski's inequality.

Corollary 3.A. Let $0<p<+\infty$. Let $u, r$ and $a$ be as in Theorem 3. A. Then

$$
\left\|S_{a, r}(u)\right\|_{L^{p}} \leq C\left\|S_{0, r}(u)\right\|_{L^{p}},
$$

where $C$ is a constant depending only on $r, p$, $a$ and $n$.

Proof. Take $q \in(0, \min (p, 2))$. By the continuous version of the vector maximal theorem of $C$. Fefferman and Stein [5] we get

$$
\begin{aligned}
& \left\{\int_{R^{n}}\left(\int_{0}^{+\infty} M_{q}(u(\cdot, t))(x)^{2} t^{2 r-1} d t\right)^{p / 2} d x\right\}^{1 / p} \\
& \quad=\left\{\int_{R^{n}}\left(\int_{0}^{+\infty} M_{1}\left(|u(\cdot, t)|^{q}\right)(x)^{2 / q} t^{2 r-1} d t\right)^{(q / 2)(p / q)} d x\right\}^{(q / p) / q} \\
& \quad \leq C\left\{\int_{R^{n}}\left(\int_{0}^{+\infty}|u(x, t)|^{q(2 / q)} t^{2 r-1} d t\right)^{(q / 2)(p / q)} d x\right\}^{(q / p) / q} \\
& \quad=C\left\|S_{0, r}(u)\right\|_{L^{p}} .
\end{aligned}
$$

Combining Theorem 3.A and the inequality (3.3) we get Corollary 3.A. 
The above clever idea to use the result of [5] is in R. Fefferman and Stein [7].

Corollary 3.B. Let $u, a$ and $p$ be as in Corollary 3. A. Then

$$
\left\|S_{a, 1}\left(D_{t} u\right)\right\|_{L^{p}} \leq C\left\|S_{0,1}\left(D_{t} u\right)\right\|_{L^{p}}
$$

and

$$
\left\|S_{a, 1}\left(D_{x_{i}} u\right)\right\|_{L^{p}} \leq C\left\|S_{0,1}\left(D_{x_{i}} u\right)\right\|_{L^{p}}, \quad i=1, \ldots, n,
$$

where $C$ is a constant depending only on $p, a$ and $n$.

Since $D_{t} u$ and $D_{x_{i}} u$ are harmonic, Corollary 3.B is a direct consequence of Corollary 3.A. Corollary 3.B means that the integrals of the $p$ th powers of $g$-functions dominate those of $S$-functions.

The argument we have explained so far was suggested by R. Fefferman. In the following part of this section, replacing the inequality (1.1) in the above argument by Theorems 1.1 and 1.2, we extend the above results to the functions of the form $u(y, t)=f *(\varphi)_{t}(y)$, which are no longer harmonic. We define

$$
S_{a, r}(f *(\kappa):)(x)
$$

by the formulae (3.1) and (3.2) with

$$
u(y, t)=f *(\kappa)_{t}(y) \text {. }
$$

(Two dots : mean two variables $y$ and $t$.) Our results are the following.

THEOREM 3.1. Let $0<\beta<\alpha$ and $-1<r<\beta$. Let $\varphi$ satisfy the condition (I. $\alpha)$. Let $\kappa \in \mathscr{B}_{\beta}^{\prime}\left(R^{n}\right), q>n /(n+\beta-r), a \geq 0, f \in$ $\bigcup_{1 \leq p \leq+\infty} L^{p}\left(R^{n}\right)$ and $x \in R^{n}$. Then

$$
S_{a, r}(f *(\kappa):)(x)^{2} \leq C \int_{0}^{+\infty} M_{q}\left(f *(\varphi)_{t}(\cdot)\right)(x)^{2} t^{2 r-1} d t,
$$

where $C$ is a constant depending only on $\alpha, \mathscr{F} \varphi(0), \beta, r, q$, a and $n$.

CoRollary 3.1. Let $\alpha, \beta, r, \varphi, \kappa, a$ and $f$ be as in Theorem 3.1. Let $p \in(n /(n+\beta-r),+\infty)$. Then

$$
\left\|S_{a, r}(f *(\kappa):)\right\|_{L^{p}} \leq C\left\|S_{0, r}(f *(\varphi):)\right\|_{L^{p}},
$$

where $C$ is a constant depending only on $\alpha, \mathscr{F} \varphi(0), \beta, r, p, a$ and $n$.

Corollary 3.2. Let $1<\beta<\alpha$. Let $\varphi$, $\kappa$ and $a$ be as in Theorem 3.1. Let $p \in(n /(n+\beta-1),+\infty)$ and let $f \in \mathscr{S}\left(R^{n}\right)$. Then

$$
\left\|S_{a, 1}\left(D_{x_{i}}(f *(\kappa):)\right)\right\|_{L^{p}} \leq C\left\|S_{0,1}\left(D_{x_{i}}(f *(\varphi):)\right)\right\|_{L^{p}}, \quad i=1, \ldots, n,
$$

where $C$ is a constant depending only on $\alpha, \mathscr{F} \varphi(0), \beta, p$, a and $n$. 
REMARK 3.1. By Remark 1.1 we can substitute $\kappa=\varphi$ in the above three results.

REMARK 3.2. If $r>-1$ and if $u(y, t)$ is a harmonic function defined by $f * P(\cdot, t)(y)$ with $f \in \bigcup_{1 \leq p \leq+\infty} L^{p}\left(R^{n}\right)$, then Theorem 3.A follows from Theorem 3.1 by substituting

$$
\varphi(x)=\kappa(x)=P(x, 1)
$$

and by taking $\alpha$ and $\beta$ so that $\max (0, r, r-n+n / q)<\beta<\alpha$, where $P(x, t)$ is the Poisson kernel in Example 1.1. Similarly, if $r>-1$ and if $u(y, t)=f * P(\cdot, t)(y)$ with $f \in \bigcup_{1 \leq p \leq+\infty} L^{p}\left(R^{n}\right)$, then Corollary 3.A follows from Corollary 3.1. If $u(y, t)=f * P(\cdot, t)(y)$ with $f \in \mathscr{S}\left(R^{n}\right)$, then the latter half of Corollary 3.B follows from Corollary 3.2.

THEOREM 3.2. Let $0<\beta<\alpha$ and $-1<r<\beta$. Let $\left\{\varphi_{1}, \ldots, \varphi_{N}\right\}$ satisfy the condition (II. $\alpha$ ). Let $\kappa \in \mathscr{B}_{\beta}^{0 \prime}\left(R^{n}\right), q>n /(n+\beta-r), a \geq 0, f \in$ $\cup_{1 \leq p \leq+\infty} L^{p}\left(R^{n}\right)$ and $x \in R^{n}$. Then

$$
S_{a, r}(f *(\kappa):)(x)^{2} \leq C \sum_{i=1}^{N} \int_{0}^{+\infty} M_{q}\left(f *\left(\varphi_{i}\right)_{t}(\cdot)\right)(x)^{2} t^{2 r-1} d t,
$$

where $C$ is a constant depending only on $\alpha, \delta, \beta, r, q, a, N$ and $n$. (For the definition of $\delta$ recall (1.3).)

COROllary 3.3. Let $\alpha, \beta, r,\left\{\varphi_{1}, \ldots, \varphi_{N}\right\}, \kappa, a$ and $f$ be as in Theorem 3.2. Let $p \in(n /(n+\beta-r),+\infty)$. Then

$$
\left\|S_{a, r}(f *(\kappa):)\right\|_{L^{p}} \leq C \sum_{i=1}^{N}\left\|S_{0, r}\left(f *\left(\varphi_{i}\right):\right)\right\|_{L^{p}},
$$

where $C$ is a constant depending only on $\alpha, \delta, \beta, r, p, a, N$ and $n$.

COROllaRY 3.4. Let $\alpha>0$. Let $\left\{\varphi_{1}, \ldots, \varphi_{N}\right\}$ satisfy the condition

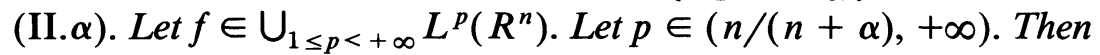

$$
\|f\|_{H^{p}} \leq C \sum_{i=1}^{N}\left\|S_{0,0}\left(f *\left(\varphi_{i}\right):\right)\right\|_{L^{p}}
$$

where $C$ is a constant depending only on $\alpha, \delta, p, N$ and $n$.

REMARK 3.3. In Corollary $3.4\|f\|_{H^{p}}$ means

$$
\left(\int_{R^{n}} \sup _{t>0}|f * P(\cdot, t)(x)|^{p} d x\right)^{1 / p} \text {. }
$$

There are several characterizations of $H^{p}\left(R^{n}\right)$. See [6] and [2] for details. 
REMARK 3.4. If $\left\{\varphi_{1}, \ldots, \varphi_{N}\right\}$ is as in Example 1.3 or 1.4 , if $f$ is as in Corollary 3.4 and if $p \in(0,+\infty)$, then the inequality (3.6) had been shown by [6] p. 172 Remark.

REMARK 3.5. If $\left\{\varphi_{1}, \ldots, \varphi_{N}\right\}$ is as in Example 1.5, $p \in(0,+\infty)$ and if $f \in H^{p}\left(R^{n}\right)$, then the inequality

$$
\|f\|_{H^{p}} \leq \sum_{i=1}^{N}\left\|S_{1,0}\left(f *\left(\varphi_{i}\right):\right)\right\|_{L^{p}}
$$

had been shown by Calderón and Torchinsky [1] and the inequality (3.6) had been shown by [14].

REMARK 3.6. The converse inequality of (3.6) (with another constant $C>0)$ is known to hold for $p \in(n /(n+\alpha),+\infty)$. Let $\mathscr{H}=$ $L_{d t / t}^{2}(0,+\infty)$. Let $T$ be the operator that assigns $\left(\varphi_{i}\right)_{t} * f(x) \in L^{2}\left(R^{n}, \mathscr{H}\right)$ to $f \in L^{2}\left(R^{n}\right)$. If $p \in(1,+\infty)$, then the argument of Hilbert space valued singular integral operators ([9] p. 83) gives $\|T f\|_{L^{p}\left(R^{n}, \mathscr{H}\right)} \leq C\|f\|_{L^{p}\left(R^{n}\right)}$ provided $f \in L^{2}\left(R^{n}\right) \cap L^{p}\left(R^{n}\right)$. If $p \in(n /(n+\alpha), 1]$, then it is easy to show that $\|T f\|_{L^{p}\left(R^{n}, \mathscr{H}\right)} \leq C\|f\|_{H^{p}\left(R^{n}\right)}$ provided $f$ is a $p$-atom. (For the definition of $p$-atoms see [2].) The converse inequality of (3.6) follows from the above two inequalities and Fatou's lemma.

From now we prove the above results. For the proof of Theorem 3.1 we need the following.

Lemma 3.1. Let $0<\beta<\alpha, 0<\varepsilon<1$ and $n /(n+\beta+1-\varepsilon)<q \leq$ 1. Let $\varphi, \kappa$ and $f$ be as in Theorem 3.1. Then

$$
\begin{aligned}
& \left|\int_{R^{n}} f(x) \kappa(x) d x\right| \\
& \quad \leq C\left(\int_{0}^{+\infty} M_{q}\left(f *(\varphi)_{t}(\cdot)\right)(0)^{q} h_{\beta, q, \varepsilon}(t) \frac{d t}{t}\right)^{1 / q},
\end{aligned}
$$

where

$$
h_{\beta, q, \varepsilon}(t)=t^{\beta q+n q-n}(1+t)^{(-n-\beta-1+\varepsilon) q+n}
$$

and where $C$ is a constant depending only on $\alpha, \mathscr{F} \varphi(0), \beta, \varepsilon, q$ and $n$.

Proof. By Theorem 1.1 it is enough to show that the right-hand side of (1.5) is bounded by the right-hand side of (3.7). 
Since $k_{\beta, \varepsilon}(y, t)$ in (1.6) is radial and decreasing as a function of $y$ variable for each fixed $t>0$, we get

$$
\begin{aligned}
\int_{R^{n}}\left|f *(\varphi)_{t}(y)\right|^{q} k_{\beta, \varepsilon}(y, t)^{q} d y \\
\leq M_{q}\left(f *(\varphi)_{t}(\cdot)\right)(0)^{q} \int k_{\beta, \varepsilon}(y, t)^{q} d y \\
\leq C M_{q}(\cdots)(0)^{q} t^{\beta q}(1+t)^{(-n-\beta-1+\varepsilon) q+n} \\
\quad=C M_{q}(\cdots)(0)^{q} t^{-n q+n} h_{\beta, q, \varepsilon}(t) .
\end{aligned}
$$

Hence the right-hand side of (1.5) is bounded by the right-hand side of (3.7).

Proof of Theorem 3.1. We may assume $x=0$ and $q \leq 1$. First we give a proof for the case $a=0$. Applying Lemma 3.1 with $\varepsilon \in(0, \min (1,1+r))$ and with dilation we get

$$
\begin{aligned}
& S_{0, r}(f *(\kappa):)(0)^{2}=\int_{0}^{+\infty}\left|f *(\kappa)_{t}(0)\right|^{2} t^{2 r-1} d t \\
& \quad \leq C \int_{0}^{+\infty} t^{2 r-1} d t\left(\int_{0}^{+\infty} M_{q}\left(f *(\varphi)_{s t}(\cdot)\right)(0)^{q} h_{\beta, q, \varepsilon}(s) \frac{d s}{s}\right)^{2 / q} \\
& \quad \leq C\left\{\int_{0}^{+\infty} h_{\beta, q, \varepsilon}(s) \frac{d s}{s}\left(\int_{0}^{+\infty} M_{q}\left(f *(\varphi)_{s t}(\cdot)\right)(0)^{2} t^{2 r-1} d t\right)^{q / 2}\right\}^{2 / q} \\
& \quad \text { by Minkowski's inequality } \\
& \quad=C\left(\int_{0}^{+\infty} h_{\beta, q, \varepsilon}(s) s^{-r q-1} d s\right)^{2 / q} \int_{0}^{+\infty} M_{q}\left(f *(\varphi)_{t}(\cdot)\right)(0)^{2} t^{2 r-1} d t .
\end{aligned}
$$

Since

$$
\int_{0}^{+\infty} h_{\beta, q, \varepsilon}(s) s^{-r q-1} d s<+\infty
$$

by $q>n /(n+\beta-r)$ and by $\varepsilon<1+r$, we get (3.4) for the case $a=0$ and $x=0$.

Next we show the case $a>0$. Put $\tau_{z} \kappa(y)=\kappa(y+z)$. Then

$$
S_{a, r}(f *(\kappa):)(0)^{2}=a^{-n} \int_{B(0, a)} S_{0, r}\left(f *\left(\tau_{z} \kappa\right):\right)(0)^{2} d z .
$$

If $z \in B(0, a)$ and if $c>0$ is small enough depending on $a, \beta$ and $n$, then $c \tau_{z} \kappa \in \mathscr{B}_{\beta}^{\prime}\left(R^{n}\right)$. Thus by the result of the case $a=0$ we get

$$
S_{0, r}\left(f *\left(\tau_{z} \kappa\right):\right)(0)^{2} \leq C \int_{0}^{+\infty} M_{q}\left(f *(\varphi)_{t}(\cdot)\right)(0)^{2} t^{2 r-1} d t
$$

Substituting (3.10) into (3.9) we get (3.4) for the case $a>0$ and $x=0$. 
The way Corollary 3.1 follows from Theorem 3.1 is the same with the way Corollary 3.A followed from Theorem 3.A. Corollary 3.2 is obtained by applying Corollary 3.1 not to the function $f$ but to the function $D_{x_{1}} f$.

For the proof of Theorem 3.2 we need the following Lemma 3.2, which can be proved in exactly the same way with Lemma 3.1 just by replacing Theorem 1.1 by Theorem 1.2. We omit the proof.

LEMMA 3.2. Let $0<\beta<\alpha, 0<\varepsilon<1$ and $n /(n+\beta+1-\varepsilon)<q \leq$ 1. Let $\left\{\varphi_{1}, \ldots, \varphi_{N}\right\}, \kappa$ and $f$ be as in Theorem 3.2. Then

$$
\left|\int f(x) \kappa(x) d x\right| \leq C \sum_{i=1}^{N}\left(\int_{0}^{+\infty} M_{q}\left(f *\left(\varphi_{i}\right)_{t}(\cdot)\right)(0)^{q} h_{\beta, q, \varepsilon}(t) \frac{d t}{t}\right)^{1 / q},
$$

where $h_{\beta, q, \varepsilon}$ is defined by (3.8) and where $C$ is a constant depending only on $\alpha, \delta, \beta, \varepsilon, q, N$ and $n$.

The way Theorem 3.2 follows from Lemma 3.2 is the same with the way Theorem 3.1 followed from Lemma 3.1. The way Corollary 3.3 follows from Theorem 3.2 is the same with the way Corollary 3.A followed from Theorem 3.A.

Proof of Corollary 3.4. Put

$$
\kappa(x)=D_{t} P(x, 1),
$$

where $P(x, t)$ is the Poisson kernel. By the result of [6] we have

$$
\left\|S_{a, 0}(f *(\kappa):)\right\|_{L^{p}}=\left\|S_{a, 1}\left(D_{t}(f * P(\cdot, \cdot))\right)\right\|_{L^{p}} \geq c\|f\|_{H^{p}} .
$$

(Recall Remark 3.4.) Thus, putting $r=0$, taking $\beta \in(\max (0, n / p-n), \alpha)$ and applying Corollary 3.3 to the above $\kappa$ we get (3.6).

\section{Proof of Theorem 1.2.}

Lemma 4.1. Let $0<\beta<\alpha$ and $0<\varepsilon<1$. Let $\left\{\varphi_{1}(x), \ldots, \varphi_{N}(x)\right\}$ satisfy the condition (II. $\alpha$ ). Let $\kappa \in \mathscr{B}_{\beta}^{0 \prime}\left(R^{n}\right)$. Let $0<a<C_{4.1}$. Let $\mathscr{E} \subset$ $R_{+}^{n+1}$ be a measurable set that satisfies

$$
\iint_{\mathscr{E} \cap Q^{\prime \prime}(x, s)} d y d t \leq a s^{n+1}
$$

for any $(x, s) \in R_{+}^{n+1}$. Then, there exist measurable functions $k_{1}(y, t), \ldots$, $k_{N}(y, t)$ defined on $R_{+}^{n+1}$ such that

$$
\left|k_{i}(y, t)\right| \leq C_{4.2} k_{\beta, \varepsilon}(y, t), \quad i=1, \ldots, N,
$$

for any $(y, t) \in R_{+}^{n+1}$ and that

$$
\kappa(x)=\iint_{\mathscr{E} c} \sum_{i=1}^{N}\left(\varphi_{i}\right)_{t}(y-x) k_{i}(y, t) d y \frac{d t}{t}
$$


for any $x \in R^{n}$, where $k_{\beta, \varepsilon}$ is defined by (1.6) and where $C_{4.1}$ and $C_{4.2}$ are positive constants depending only on $\alpha, \beta, \varepsilon, \delta, N$ and $n$. (For the definition of $Q^{\prime \prime}(x, s)$ and $\delta$ see $§ 1$.)

Since the proof of Lemma 4.1 is lengthy, we postpone it to $\$ 5$.

Accepting Lemma 4.1 temporarily we prove Theorem 1.2. For $(x, s)$ $\in R_{+}^{n+1}$ let

$$
w_{q}(x, s)=\left\{s^{-n-1} \iint_{Q^{\prime}(x, s)}\left(\sum_{i=1}^{N}\left|f *\left(\varphi_{i}\right)_{t}(y)\right|\right)^{q} d y d t\right\}^{1 / q} .
$$

Let $A>1$ and let

(4.4) $\mathscr{E}=\left\{(x, s) \in R_{+}^{n+1}:\left(\sum_{i=1}^{N}\left|f *\left(\varphi_{i}\right)_{s}(x)\right|\right)^{q}>A w_{q}(x, s)^{q}\right\}$.

Let $(y, t) \in Q^{\prime \prime}(x, s)$. Since $Q^{\prime}(y, t) \supset Q^{\prime \prime}(x, s)$, we have

(4.5) $w_{q}(y, t)^{q} \geq\left(\frac{2}{3}\right)^{n+1} s^{-n-1} \iint_{Q^{\prime \prime}(x, s)}\left(\sum_{i=1}^{N}\left|f *\left(\varphi_{i}\right)_{u}(z)\right|\right)^{q} d z d u$.

Therefore

$$
\begin{aligned}
\iint_{\mathscr{E} \cap Q^{\prime \prime}(x, s)} d y d t & \leq \iint_{Q^{\prime \prime}(x, s)}\left(\sum\left|f *\left(\varphi_{i}\right)_{t}(y)\right|\right)^{q} A^{-1} w_{q}(y, t)^{-q} d y d t \\
& \text { by the definition of } \mathscr{E} \\
& \leq(3 / 2)^{n+1} s^{n+1} A^{-1} \quad \text { by }(4.5) .
\end{aligned}
$$

So, the set $\mathscr{E}$ defined by (4.4) satisfies (4.1) with

$$
a=(3 / 2)^{n+1} A^{-1} \text {. }
$$

Let $A>1$ be so large that $(4.6)<C_{4.1}$. Applying Lemma 4.1 with (4.6) and (4.4) gives $k_{1}(y, t), \ldots, k_{N}(y, t)$ that satisfy (4.2) and (4.3). Thus

$$
\begin{aligned}
& \left|\int_{R^{n}} f(x) \kappa(x) d x\right| \\
& \quad=\left|\int_{R^{n}} f(x) d x \iint_{\mathscr{E}^{c}} \sum_{i=1}^{N}\left(\varphi_{i}\right)_{t}(y-x) k_{i}(y, t) d y \frac{d t}{t}\right| \quad \text { by }(4.3) \\
& \quad=\left|\iint_{\mathscr{E}^{c}} \sum_{i} f *\left(\varphi_{i}\right)_{t}(y) k_{i}(y, t) d y \frac{d t}{t}\right| \quad \text { by } f \in \bigcup_{1 \leq p \leq+\infty} L^{p}\left(R^{n}\right) \\
& \quad \leq \iint_{R_{+}^{n+1}} A^{1 / q_{w_{q}}}(y, t) C k_{\beta, \varepsilon}(y, t) d y \frac{d t}{t}
\end{aligned}
$$

by (4.2) and by the definition of $\mathscr{E}$. 
Put $\omega=n^{-1 / 2}$. For $(y, t) \in R_{+}^{n+1}$ let

$$
S(y, t)=\left\{\left(j_{1}, \ldots, j_{n}, i\right) \in Z^{n+1}: t / 2 \leq 2^{i} \leq 2 t,\left|y-\omega 2^{i} j\right| \leq 2^{i+1}\right\},
$$

where $Z$ is the set of all integers and where $j=\left(j_{1}, \ldots, j_{n}\right) \in Z^{n}$. Note that

$$
Q^{\prime}(y, t) \subset \bigcup_{(j, i) \in S(y, t)} Q^{\prime}\left(\omega 2^{i} j, 2^{i}\right)
$$

and that if $(j, i) \in S(y, t)$, then

$$
c k_{\beta, \varepsilon}(y, t) \leq k_{\beta, \varepsilon}\left(\omega 2^{i} j, 2^{i}\right) \leq C k_{\beta, \varepsilon}(y, t) .
$$

So

(4.8) $w_{q}(y, t) k_{\beta, \varepsilon}(y, t) \leq C \sum_{(j, i) \in S(y, t)} w_{q}\left(\omega 2^{i} j, 2^{i}\right) k_{\beta, \varepsilon}\left(\omega 2^{i} j, 2^{i}\right)$.

Note that

$$
\iint_{\left\{(y, t) \in R_{+}^{n+1}: S(y, t) \ni\left(\omega 2^{\prime} j, 2^{\prime}\right)\right\}} d y \frac{d t}{t} \leq C 2^{i n}
$$

for any $(j, i) \in Z^{n+1}$ and that

$$
\left\|\sum_{(j, i) \in Z^{n+1}} \chi_{Q^{\prime}\left(\omega 2^{i} j, 2^{l}\right)}\right\|_{L^{\infty}} \leq C .
$$

Therefore,

$$
\begin{aligned}
(4.7) & \leq C A^{1 / q} \iint_{R_{+}^{n+1}} \sum_{(j, i) \in S(y, t)} w_{q}\left(\omega 2^{i} j, 2^{i}\right) k_{\beta, \varepsilon}\left(\omega 2^{i} j, 2^{i}\right) d y \frac{d t}{t} \\
& \leq C A^{1 / q} \sum_{(j, i) \in Z^{n+1}} w_{q}\left(\omega 2^{i} j, 2^{i}\right) k_{\beta, \varepsilon}\left(\omega 2^{i} j, 2^{i}\right) 2^{i n} \quad \text { by } q \leq 1 \\
& \leq C A^{1 / q}\left(\sum_{(j, i) \in Z^{n+1}} w_{q}(\cdots)^{q} k_{\beta, \varepsilon}(\cdots)^{q} 2^{i n q}\right)^{1 / q} \text { by }(4.9) \\
& \leq C A^{1 / q}\left(\iint_{R_{+}^{n+1}}\left(\sum_{i=1}^{N}\left|f *\left(\varphi_{i}\right)_{t}(y)\right|\right)^{q} k_{\beta, \varepsilon}(y, t)^{q} t^{n q} t^{-n-1} d y d t\right)^{1 / q}
\end{aligned}
$$

which implies Theorem 1.2. 


\section{Proof of Lemma 4.1.}

LEMMA 5.1. Let $a>0$ and let $\mathscr{E} \subset R_{+}^{n+1}$ be a measurable set that satisfies (4.1) for any $(x, s) \in R_{+}^{n+1}$. Let $b \geq 1$ and let $K(y, t)$ be a positive measurable function defined on $R_{+}^{n+1}$ such that

$$
K(y, t) \leq b K(x, s)
$$

for any $(x, s) \in R_{+}^{n+1}$ and any $(y, t) \in Q^{\prime}(x, s)$. Let $t_{0}>0$. Let

$$
T=Q\left(0, t_{0}\right) \quad \text { or } \quad R_{+}^{n+1} \backslash Q\left(0, t_{0}\right) \quad \text { or } \quad Q\left(0,2 t_{0}\right) \backslash Q\left(0, t_{0}\right) .
$$

Then

$$
\iint_{\mathscr{E} \cap T} K(y, t) d y d t \leq C b a \iint_{T} K(y, t) d y d t,
$$

where $C$ is a constant depending only on $n$.

Proof. Let $\left\{\left(y_{i}, t_{i}\right)\right\}_{i=1}^{\infty} \subset R_{+}^{n+1}$ be maximal with respect to the properties

$$
\left(y_{i}, t_{i}\right) \in T \quad \text { and } \quad\left|y_{i}-y_{j}\right|+\left|t_{i}-t_{j}\right| \geq 0.01\left(t_{i}+t_{j}\right) \quad \text { if } i \neq j .
$$

Then geometric observation gives

$$
T \subset \bigcup_{i=1}^{\infty} Q^{\prime \prime}\left(y_{i}, t_{i}\right)
$$

$$
\iint_{Q^{\prime \prime}\left(y_{i}, t_{i}\right)} d y d t \leq C \iint_{T \cap Q^{\prime \prime}\left(y_{i}, t_{i}\right)} d y d t
$$

and

$$
\left\|\sum_{i=1}^{\infty} \chi_{Q^{\prime \prime}\left(y_{i}, t_{i}\right)}\right\|_{L^{\infty}} \leq C,
$$

where the constant $C$ in (5.3)-(5.4) depends only on $n$. Then

$$
\begin{array}{rl}
\iint_{\mathscr{E} \cap T} & K(y, t) d y d t \\
& \leq \sum_{i=1}^{\infty} \iint_{\mathscr{E} \cap T \cap Q^{\prime \prime}\left(y_{i}, t_{i}\right)} K(y, t) d y d t \quad \text { by }(5.2) \\
& \leq C b \sum \iint_{\mathscr{E} \cap T \cap Q^{\prime \prime}\left(y_{i}, t_{i}\right)} d y d t \quad \inf _{(x, s) \in Q^{\prime \prime}\left(y_{i}, t_{i}\right)} K(x, s) \quad \text { by }(5.1) \\
& \leq C b a \sum \iint_{T \cap Q^{\prime \prime}\left(y_{t}, t_{i}\right)} K(y, t) d y d t \quad \text { by }(4.1) \text { and }(5.3) \\
& \leq C b a \iint_{T} K(y, t) d y d t \quad \text { by }(5.4),
\end{array}
$$

which implies the desired result. 
We can extend Lemma 5.1 to more general $T$, but we do not need it in the sequel.

Lemma 5.2. Let $0<\beta<\alpha$ and $0<\varepsilon^{\prime}<\varepsilon<1$. Let $a>0$ and let $\mathscr{E} \subset R_{+}^{n+1}$ be a measurable set that satisfies (4.1) for any $(x, s) \in R_{+}^{n+1}$. Let $(y, t) \in R_{+}^{n+1}$. Then

$$
\iint_{\mathscr{E}} k_{\beta, \varepsilon}(x, s) s^{-n} k_{\alpha, \varepsilon^{\prime}}\left(\frac{y-x}{s}, \frac{t}{s}\right) d x \frac{d s}{s} \leq C a k_{\beta, \varepsilon}(y, t),
$$

where $k_{\beta, \varepsilon}$ is defined by (1.6) and where $C$ is a constant depending only on $\alpha, \beta, \varepsilon, \varepsilon^{\prime}$ and $n$.

Proof. Put

$u(y, t)=$ the left-hand side of $(5.5)$

$$
=t^{\alpha} \iint_{\mathscr{E}} s^{\beta-\varepsilon^{\prime}}(1+|x|+s)^{-n-\beta-1+\varepsilon}(|y-x|+t+s)^{-n-\alpha-1+\varepsilon^{\prime}} d x d s .
$$

If $(y, t) \in Q(0,1)$, then

$$
\begin{aligned}
u(y, t) \leq & t^{\alpha} \iint_{\mathscr{E} \cap Q(0,2)} s^{\beta-\varepsilon^{\prime}}(|y-x|+t+s)^{-n-\alpha-1+\varepsilon^{\prime}} d x d s \\
& +C t^{\alpha} \iint_{\mathscr{E} \cap Q(0,2)^{c^{c}}} s^{\beta-\varepsilon^{\prime}}(|x|+s)^{-2 n-2-\alpha-\beta+\varepsilon+\varepsilon^{\prime}} d x d s \\
\leq & \operatorname{Cat}^{\alpha} \iint_{Q(0,2)} s^{\beta-\varepsilon^{\prime}}(|y-x|+t+s)^{-n-\alpha-1+\varepsilon^{\prime}} d x d s \\
& +\operatorname{Cat}^{\alpha} \iint_{R_{+}^{n+1} \backslash Q(0,2)} s^{\beta-\varepsilon^{\prime}}(|x|+s)^{-2 n-2-\alpha-\beta+\varepsilon+\varepsilon^{\prime}} d x d s
\end{aligned}
$$

by Lemma 5.1

$$
\begin{aligned}
& \leq \operatorname{Cat}^{\alpha} \int_{0}^{2} s^{\beta-\varepsilon^{\prime}}(t+s)^{-\alpha-1+\varepsilon^{\prime}} d s \\
& \quad+\operatorname{Cat}^{\alpha} \int_{0}^{+\infty} s^{\beta-\varepsilon^{\prime}}(1+s)^{-n-2-\alpha-\beta+\varepsilon+\varepsilon^{\prime}} d s \\
& \leq C^{\beta} t^{\beta} \text { by } \beta<\alpha .
\end{aligned}
$$


If $(y, t) \notin Q(0,1)$ and if $t>|y|$, then

$$
\begin{aligned}
u(y, t) \leq & t^{\alpha} \iint_{\mathscr{E} \cap Q(0,2 t)} s^{\beta-\varepsilon^{\prime}}(1+|x|+s)^{-n-\beta-1+\varepsilon} t^{-n-\alpha-1+\varepsilon^{\prime}} d x d s \\
& +C t^{\alpha} \iint_{\mathscr{E} \cap Q(0,2 t)^{c}} s^{\beta-\varepsilon^{\prime}}(|x|+s)^{-2 n-2-\alpha-\beta+\varepsilon+\varepsilon^{\prime}} d x d s \\
\leq & C a t^{-n-1+\varepsilon^{\prime}} \int_{0}^{2 t} s^{\beta-\varepsilon^{\prime}}(1+s)^{-\beta-1+\varepsilon} d s \\
& +\operatorname{Cat}^{\alpha} \int_{0}^{+\infty} s^{\beta-\varepsilon^{\prime}}(t+s)^{-n-2-\alpha-\beta+\varepsilon+\varepsilon^{\prime}} d s \quad \text { by Lemma } 5.1 \\
\leq & \text { Cat }^{-n-1+\varepsilon} \text { by } \varepsilon^{\prime}<\varepsilon .
\end{aligned}
$$

If $(y, t) \notin Q(0,1)$ and if $t \leq|y|$, then

$$
\begin{aligned}
u(y, t) \leq & C t^{\alpha} \iint_{\mathscr{E} \cap Q(0,|y| / 2)} s^{\beta-\varepsilon^{\prime}}(1+|x|+s)^{-n-\beta-1+\varepsilon}|y|^{-n-\alpha-1+\varepsilon^{\prime}} d x d s \\
& +C t^{\alpha} \iint_{\mathscr{E} \cap(Q(0,2|y|) \backslash Q(0,|y| / 2))} s^{\beta-\varepsilon^{\prime}|y|^{-n-\beta-1+\varepsilon}} \times(|y-x|+t+s)^{-n-\alpha-1+\varepsilon^{\prime}} d x d s \\
& +C t^{\alpha} \iint_{\mathscr{E} \cap Q(0,2 \mid y)^{c}} s^{\beta-\varepsilon^{\prime}}(|x|+s)^{-2 n-2-\alpha-\beta+\varepsilon+\varepsilon^{\prime}} d x d s \\
\leq & C a t^{\alpha}|y|^{-n-\alpha-1+\varepsilon^{\prime}} \int_{0}^{|y| / 2} s^{\beta-\varepsilon^{\prime}}(1+s)^{-\beta-1+\varepsilon} d s \\
& +C a t^{\alpha}|y|^{-n-\beta-1+\varepsilon} \int_{0}^{2|y|} s^{\beta-\varepsilon^{\prime}}(t+s)^{-\alpha-1+\varepsilon^{\prime}} d s \\
& +C^{\alpha} \int_{0}^{+\infty} s^{\beta-\varepsilon^{\prime}}(|y|+s)^{-n-2-\alpha-\beta+\varepsilon+\varepsilon^{\prime}} d s \text { by Lemma 5.1 } \\
\leq & C a t^{\beta}|y|^{-n-\beta-1+\varepsilon} \text { by } \beta<\alpha \text { and } \varepsilon^{\prime}<\varepsilon .
\end{aligned}
$$

Thus in all cases we get $u(y, t) \leq \operatorname{Cak}_{\beta, \varepsilon}(y, t)$.

LEMMA 5.3. Let $\alpha>0$. Let $\left\{\varphi_{1}, \ldots, \varphi_{N}\right\}$ satisfy the condition (II. $\alpha$ ). Then there exist $\psi_{1}, \ldots, \psi_{N} \in L^{2}\left(R^{n}\right) \cap C^{\infty}\left(R^{n}\right)$ such that

$$
\int_{0}^{+\infty} \sum_{i=1}^{N} \mathscr{F} \varphi_{i}(t \xi) \mathscr{F} \psi_{i}(t \xi) \frac{d t}{t}=1
$$

for any $\xi \neq 0$,

$$
\operatorname{supp} \mathscr{F} \psi_{i} \not \supset 0, \quad i=1, \ldots, N
$$


and that

$$
\left|D_{x}^{\gamma} \psi_{i}(x)\right| \leq C(1+|x|)^{-n-[\alpha]-2}, \quad i=1, \ldots, N,
$$

for any multi-index $\gamma$ with $l(\gamma) \leq 1$, where $C$ is a constant depending only on $\alpha, \delta, N$ and $n$.

Proof. Let $\varepsilon \in(0,1)$. Let $\theta \in C^{\infty}(0,+\infty)$ be a nonnegative function such that $\theta(t)=1$ if $t \in(\varepsilon, 1 / \varepsilon)$ and that $\operatorname{supp} \theta \subset(\varepsilon / 2,2 / \varepsilon)$. By (II. $\alpha .1)$ and (II. $\alpha .2)$ we have $\left|\mathscr{F} \varphi_{i}(\xi)\right| \leq \min \left(|\xi|, C|\xi|^{-\alpha}\right)$ and $\left|D_{\xi}^{\gamma} \mathscr{F} \varphi_{i}(\xi)\right| \leq 1$ if $l(\gamma)=1$. Hence if $\varepsilon>0$ is small enough depending on $\alpha, \delta, N$ and $n$, then

$$
\inf _{|\xi|=1} \int_{0}^{+\infty} \sum_{i=1}^{N}\left|\mathscr{F} \varphi_{i}(t \xi)\right|^{2} \theta(t) \frac{d t}{t}>c_{\alpha, \delta, N, n}>0 .
$$

For this small $\varepsilon$ take $\psi_{1}, \ldots, \psi_{N} \in L^{2}\left(R^{n}\right)$ so that

$$
\begin{aligned}
\mathscr{F} \psi_{i}(\xi)=\overline{\mathscr{F} \varphi_{i}(\xi)} \theta(|\xi|)\left\{\int_{0}^{+\infty} \sum_{j=1}^{N}\left|\mathscr{F} \varphi_{j}\left(\frac{t \xi}{|\xi|}\right)\right|^{2} \theta(t) \frac{d t}{t}\right\}^{-1}, \\
i=1, \ldots, N .
\end{aligned}
$$

(This is due to [1].) Then (5.6)-(5.7) are easy. (5.8) follows from (II. $\alpha .2)$.

REMARK 5.1. Similarly we can show that if $\varphi$ satisfies (I. $\alpha$ ), then there exists $\psi \in L^{2}\left(R^{n}\right) \cap C^{\infty}\left(R^{n}\right)$ satisfying

$$
\int_{0}^{+\infty} \mathscr{F} \varphi(t \xi) \mathscr{F} \psi(t \xi) \frac{d t}{t}=1 \text { for any } \xi \neq 0,
$$

$$
\operatorname{supp} \mathscr{F} \psi \not \nexists 0
$$

and

(5.8) $\left|D_{x}^{\gamma} \psi(x)\right| \leq C(1+|x|)^{-n-[\alpha]-2}$ for any $\gamma$ with $l(\gamma) \leq 1$, where $C$ is a constant depending only on $\alpha, \mathscr{F} \varphi(0)$ and $n$.

LEMMA 5.4. Let $\alpha>0$. Let $\psi \in L^{2}\left(R^{n}\right) \cap C^{\infty}\left(R^{n}\right)$ satisfy (5.7)' and $(5.8)^{\prime \prime} \quad\left|D_{x}^{\gamma} \psi(x)\right| \leq(1+|x|)^{-n-[\alpha]-2}$ for any $\gamma$ with $l(\gamma) \leq 1$.

Then, there exists a sequence of functions $\left\{v_{i}\right\}_{i=0}^{\infty} \subset L^{\infty}\left(R^{n}\right)$ such that

$$
\psi(x)=\sum_{i=0}^{\infty} 2^{-i([\alpha]+2)}\left(v_{i}\right)_{2^{i}}(x),
$$

$$
\operatorname{supp} v_{i} \subset B(0,1) \text {, }
$$

$$
\left\|v_{i}\right\|_{L^{\infty}} \leq C
$$


and

$$
\int v_{i}(x) x^{\gamma} d x=0
$$

for any multi-index $\gamma$ with $l(\gamma) \leq[\alpha]+1$, where $C$ is a constant depending only on $\alpha$ and $n$.

Proof. Let $h \in \mathscr{S}\left(R^{n}\right)$ be a nonnegative function such that

$$
\text { supp } h \subset B(0,1) \backslash B(0,1 / 4)
$$

and that

$$
\sum_{j=1}^{\infty} h\left(2^{-j} x\right)=1 \quad \text { if }|x|>1
$$

Let $\left\{\pi_{j}(x)\right\}_{j=1}^{L}$ be an orthonormal basis for the Hilbert space of polynomials of degree $\leq[\alpha]+1$ with norm

$$
\|P\|=\left(\int|P(x)|^{2} h(x) d x\right)^{1 / 2} \text {. }
$$

Put

$$
\begin{aligned}
\psi(x) & =\left(1-\sum_{i=1}^{\infty} h\left(2^{-i} x\right)\right) \psi(x)+\sum_{i=1}^{\infty} h\left(2^{-i} x\right) \psi(x) \\
& =\theta_{0}(x)+\sum_{i=1}^{\infty} \theta_{i}(x)
\end{aligned}
$$

and

$$
\zeta_{i}(x)=2^{-i n} \sum_{j=1}^{L} \int \sum_{k=i+1}^{\infty} \theta_{k}(y) \pi_{j}\left(2^{-i} y\right) d y \pi_{j}\left(2^{-i} x\right) h\left(2^{-i} x\right)
$$

Note that by $(5.8)^{\prime \prime}$ and by $\operatorname{deg} \pi_{j} \leq[\alpha]+1$ the above integrands are integrable and that

$$
\left\|\zeta_{i}\right\|_{L^{\infty}} \leq C 2^{-i(n+[\alpha]+2)}
$$

Put

$$
\begin{aligned}
\psi & =\sum_{i=0}^{\infty} \theta_{i}=\left(\theta_{0}+\zeta_{0}\right)+\sum_{i=1}^{\infty}\left(\theta_{i}-\zeta_{i-1}+\zeta_{i}\right) \\
& =v_{0}+\sum_{i=1}^{\infty} 2^{-i([\alpha]+2)}\left(v_{i}\right)_{2^{i}}
\end{aligned}
$$


Then (5.10) is clear. (5.11) follows from (5.8)" and (5.13). Since $\left\{\sum_{k=i+1}^{\infty} \theta_{k}\right.$ $\left.-\zeta_{i}\right\}_{i=0}^{\infty}$ are orthogonal to all the polynomials of degree $\leq[\alpha]+1,(5.12)$ for $i \geq 1$ is easy. Since $\int \psi(x) x^{\gamma}=0$ for any $\gamma$ with $l(\gamma) \leq[\alpha]+1$ by $(5.7)^{\prime}$ and (5.8)', (5.12) holds for the case $i=0$, too.

REMARK 5.2. In a similar way with Lemma 5.4 we can show that if $\beta>0, \kappa \in \mathscr{B}_{\beta}^{\prime}\left(R^{n}\right)$ and if $\int \kappa(x) d x=0$, then $c \kappa \in \mathscr{B}_{\beta}^{0 \prime}\left(R^{n}\right)$ with $c>0$ small enough depending only on $\beta$ and $n$.

LEMMA 5.5. Let $0<\nu \leq[\alpha]+1$ and let $0<\varepsilon<1$. Let $\psi \in L^{2}\left(R^{n}\right) \cap$ $C^{\infty}\left(R^{n}\right)$ satisfy $(5.7)^{\prime}$ and $(5.8)^{\prime \prime}$. Let $\kappa \in \mathscr{B}_{\nu}^{0 \prime}\left(R^{n}\right)$. Then

$$
\left|\kappa *(\psi)_{t}(y)\right| \leq C k_{\nu, \varepsilon}(y, t),
$$

where $k_{\nu, \varepsilon}$ is defined by (1.6) and where the constant $C$ depends only on $\alpha, \nu, \varepsilon$ and $n$.

Proof. First we assume

$$
\kappa \in \mathscr{B}_{\nu}^{0}\left(R^{n}\right) \text {. }
$$

Lemma 5.4 gives $\left\{v_{i}(x)\right\}_{i=0}^{\infty}$ that satisfy (5.9)-(5.12). Thus

$$
\begin{array}{ll}
\left|\kappa *(\psi)_{t}(y)\right| \leq \sum_{i=0}^{\infty} 2^{-i([\alpha]+2)}\left|\int \kappa(y-x)\left(v_{i}\right)_{2^{i} t}(x) d x\right| & \\
\leq \sum_{i=0}^{\infty} 2^{-i([\alpha]+2)} \inf _{\operatorname{deg} P \leq \nu} \int|\kappa(y-x)-P(x)|\left|\left(v_{i}\right)_{2^{2} t}(x)\right| d x & \text { by (5.12) } \\
\leq \sum 2^{-i([\alpha]+2)}\left(2^{i} t\right)^{\nu} \leq C t^{\nu} . &
\end{array}
$$

If $(y, t) \notin Q(0,2)$, then

$$
\begin{aligned}
\left|\kappa *(\psi)_{t}(y)\right| & =\left|\int\left\{(\psi)_{t}(y-x)-(\psi)_{t}(y)\right\} \kappa(x) d x\right| \\
& \leq C \sup _{x \in B(0,1)}\left|(\psi)_{t}(y-x)-(\psi)_{t}(y)\right| \quad \text { by } \int \kappa=0 \\
& \leq C t^{-n-1}(1+|y| / t)^{-n-[\alpha]-2} \text { by }(5.8)^{\prime \prime} .
\end{aligned}
$$

Since $[\alpha]+1 \geq \nu,(5.16)$ and (5.17) imply

$$
\left|\kappa *(\psi)_{t}(y)\right| \leq C k_{\nu, 0}(y, t) .
$$


Next we remove the restriction (5.15). Let $\kappa \in \mathscr{B}_{\nu}^{0 \prime}\left(R^{n}\right)$. By its definition $\kappa$ can be decomposed into the sum $\sum_{j=0}^{\infty} 2^{-j}\left(\kappa_{j}\right)_{2^{j}}$ with $\kappa_{j} \in$ $\mathscr{B}_{\nu}^{0}\left(R^{n}\right)$. Applying (5.18) with dilation to each $\kappa_{j}$ gives

$$
\begin{aligned}
\left|2^{-j}\left(\kappa_{j}\right)_{2^{j}} *(\psi)_{t}(y)\right| & \leq C 2^{-j(n+1)} k_{\nu, 0}\left(y / 2^{j}, t / 2^{j}\right) \\
& =C t^{\nu}\left(2^{j}+|y|+t\right)^{-n-\nu-1} .
\end{aligned}
$$

Summing up (5.19) with respect to $j$ gives (5.14).

LEMMA 5.6. Let $0<\beta<\alpha$ and $0<\varepsilon^{\prime}<\varepsilon<1$. Let $\left\{\varphi_{1}, \ldots, \varphi_{N}\right\}$ satisfy (II. $\alpha$ ). Let $\psi_{1}, \ldots, \psi_{N} \in L^{2}\left(R^{n}\right) \cap C^{\infty}\left(R^{n}\right)$ satisfy (5.6)-(5.8). Let $\kappa \in \mathscr{B}_{\beta}^{0 \prime}\left(R^{n}\right)$. Let $a>0$ and let $\mathscr{E} \subset R_{+}^{n+1}$ be a measurable set that satisfies (4.1) for any $(x, s) \in R_{+}^{n+1}$. Let

$$
\begin{aligned}
k_{i, j}(y, t) & =\check{\varphi}_{i} *\left(\check{\psi}_{j}\right)_{t}(y), \\
k_{i}^{1}(y, t) & =\kappa *\left(\check{\psi}_{i}\right)_{t}(y)
\end{aligned}
$$

and

(5.22) $\quad k_{i}^{m}(y, t)=\iint_{\mathscr{E}} \sum_{j=1}^{N} k_{j}^{m-1}(x, s) s^{-n} k_{j, i}\left(\frac{y-x}{s}, \frac{t}{s}\right) d x \frac{d s}{s}$,

where $i, j \in\{1, \ldots, N\}, m \in\{2,3,4, \ldots\}$ and $(y, t) \in R_{+}^{n+1}$. Then

$$
\begin{gathered}
\left|k_{i, j}(y, t)\right| \leq C k_{\alpha, \varepsilon^{\prime}}(y, t), \\
\varphi_{i}(-x)=\iint_{R_{+}^{n+1}} \sum_{j=1}^{N}\left(\varphi_{j}\right)_{s}(z-x) k_{i, j}(z, s) d z \frac{d s}{s}, \\
\left|k_{i}^{m}(y, t)\right| \leq C(C a)^{m-1} k_{\beta, \varepsilon}(y, t)
\end{gathered}
$$

and

$$
\begin{aligned}
\kappa(x)= & \iint_{\mathscr{E}^{c}} \sum_{i=1}^{N}\left(\varphi_{i}\right)_{t}(y-x) \sum_{l=1}^{m} k_{l}^{l}(y, t) d y \frac{d t}{t} \\
& +\iint_{\mathscr{E}_{i}} \sum_{i=1}^{N}\left(\varphi_{i}\right)_{t}(y-x) k_{i}^{m}(y, t) d y \frac{d t}{t}
\end{aligned}
$$

for any $i, j \in\{1, \ldots, N\}$, any $m \in\{1,2,3, \ldots\}$, any $x \in R^{n}$ and any $(y, t) \in R_{+}^{n+1}$, where $k_{\alpha, \varepsilon^{\prime}}$ and $k_{\beta, \varepsilon}$ are defined by (1.6) and where $C$ is a constant depending only on $\alpha, \beta, \varepsilon, \varepsilon^{\prime}, \delta, N$ and $n$. 
(5.23) follows directly from Lemma 5.5 (with $\nu=\alpha$ ). (5.24) follows from (5.6).

Proof of (5.25). We prove this by induction with respect to $m$. The case $m=1$ is clear from Lemma 5.5 (with $\nu=\beta$ ). Suppose that (5.25) holds for some $m$. Then

$$
\begin{aligned}
\left|k_{i}^{m+1}(y, t)\right| & \leq \iint_{\mathscr{E}} \sum_{j=1}^{N}\left|k_{j}^{m}(x, s)\right| s^{-n}\left|k_{j, i}\left(\frac{y-x}{s}, \frac{t}{s}\right)\right| d x \frac{d s}{s} \\
& \leq \iint_{\mathscr{E}} C(C a)^{m-1} k_{\beta, \varepsilon}(x, s) s^{-n} k_{\alpha, \varepsilon^{\prime}}\left(\frac{y-x}{s}, \frac{t}{s}\right) d x \frac{d s}{s} \\
& \quad \text { by }(5.23) \text { and by the hypothesis of induction } \\
& \leq(C a)^{m} k_{\beta, \varepsilon}(y, t) \text { by Lemma 5.2. }
\end{aligned}
$$

Thus the induction is completed.

Proof of (5.26). We prove this by induction with respect to $m$. The case $m=1$ is clear from (5.6). Suppose that (5.26) holds for some $m$. Note that applying translation and dilation to (5.24) gives

$$
\left(\varphi_{l}\right)_{t}(y-x)=\iint_{R_{+}^{n+1}} \sum_{j=1}^{N}\left(\varphi_{j}\right)_{s}(z-x) t^{-n} k_{i, j}\left(\frac{z-y}{t}, \frac{s}{t}\right) d z \frac{d s}{s} .
$$

Thus, the second term on the right-hand side of (5.26)

$$
\begin{aligned}
= & \iint_{\mathscr{E}} \sum_{i=1}^{N}\left(\varphi_{i}\right)_{t}(y-x) k_{i}^{m}(y, t) d y \frac{d t}{t} \\
= & \iint_{\mathscr{E}} \sum_{i=1}^{N} k_{t}^{m}(y, t) d y \frac{d t}{t} \\
& \times \iint_{R_{+}^{n+1}} \sum_{j=1}^{N}\left(\varphi_{j}\right)_{s}(z-x) t^{-n} k_{i, j}\left(\frac{z-y}{t}, \frac{s}{t}\right) d z \frac{d s}{s} \\
= & \iint_{R_{+}^{n+1}} \sum_{j=1}^{N}\left(\varphi_{j}\right)_{s}(z-x) d z \frac{d s}{s} \\
& \times \int_{\mathscr{E}} \sum_{i=1}^{N} k_{i}^{m}(y, t) t^{-n} k_{t, j}\left(\frac{z-y}{t}, \frac{s}{t}\right) d y \frac{d t}{t} \\
= & \iint_{R_{+}^{n+1}} \sum_{j=1}^{N}\left(\varphi_{j}\right)_{s}(z-x) k_{J}^{m+1}(z, s) d z \frac{d s}{s} .
\end{aligned}
$$


Substituting this into (5.26) gives

$$
\begin{aligned}
\kappa(x)= & \iint_{\mathscr{E}^{c}} \sum_{i=1}^{N}\left(\varphi_{i}\right)_{t}(y-x) \sum_{l=1}^{m} k_{i}^{l}(y, t) d y \frac{d t}{t} \\
& +\iint_{R_{+}^{n+1}} \sum_{i=1}^{N}\left(\varphi_{i}\right)_{t}(y-x) k_{i}^{m+1}(y, t) d y \frac{d t}{t} \\
= & \iint_{\mathscr{E}^{c}} \sum_{i=1}^{N}\left(\varphi_{i}\right)_{t}(y-x) \sum_{l=1}^{m+1} k_{i}^{l}(y, t) d y \frac{d t}{t} \\
& +\iint_{\mathscr{E}} \sum_{i=1}^{N}\left(\varphi_{i}\right)_{t}(y-x) k_{i}^{m+1}(y, t) d y \frac{d t}{t} .
\end{aligned}
$$

Thus the induction is completed.

Proof of Lemma 4.1. The assumptions in Lemma 4.1 give $\alpha, \beta, \varepsilon$, $\left\{\varphi_{1}, \ldots, \varphi_{N}\right\}, \kappa, a$ and $\mathscr{E}$. Lemma 5.3 gives $\left\{\psi_{1}, \ldots, \psi_{N}\right\}$. Take $\varepsilon^{\prime} \in(0, \varepsilon)$. Then, applying Lemma 5.6 to these gives us $\left\{k_{i}^{m}(y, t)\right\}_{m=1,2,3, \ldots ; i=1, \ldots, N}$ that satisfy (5.25)-(5.26). If $C_{4.1}$ in Lemma 4.1 is small enough, then by (5.25) $\sum_{m=1}^{\infty} k_{i}^{m}(y, t)$ converges everywhere. Put

$$
k_{i}(y, t)=\sum_{m=1}^{\infty} k_{i}^{m}(y, t)
$$

Then (4.2) follows from (5.25). Since the second term on the right-hand side of (5.26) goes to zero by (5.25), (4.3) follows from (5.26).

6. Proof of Theorem 1.1. The proof of Theorem 1.1 is very similar to the proof of Theorem 1.2. All we need is the following Lemma 6.1, which corresponds to Lemma 4.1. The way Theorem 1.1 follows from Lemma 6.1 is exactly the same with the way Theorem 1.2 followed from Lemma 4.1. We do not repeat this argument.

LEMMA 6.1. Let $0<\beta<\alpha$ and $0<\varepsilon<1$. Let $\varphi$ satisfy the condition (I. $\alpha)$. Let $\kappa \in \mathscr{B}_{\beta}^{\prime}\left(R^{n}\right)$. Let $0<a<C_{6.1}$. Let $\mathscr{E} \subset R_{+}^{n+1}$ be a measurable set that satisfies (4.1) for any $(x, s) \in R_{+}^{n+1}$. Then there exists a measurable function $k(y, t)$ defined on $R_{+}^{n+1}$ such that

$$
|k(y, t)| \leq C_{6.2} k_{\beta, \varepsilon}(y, t)
$$

for any $(y, t) \in R_{+}^{n+1}$ and that

$$
\kappa(x)=\iint_{\mathscr{E}^{c}}(\varphi)_{t}(y-x) k(y, t) d y \frac{d t}{t}
$$


for any $x \in R^{n}$, where $k_{\beta, \varepsilon}$ is defined by (1.6) and where $C_{6.1}$ and $C_{6.2}$ are positive constants depending only on $\alpha, \mathscr{F} \varphi(0), \beta, \varepsilon$ and $n$.

Proof. By Remark 5.1 we get $\psi$ that satisfies $(5.6)^{\prime}-(5.8)^{\prime}$. Take $\varepsilon^{\prime} \in(0, \varepsilon)$.

Put

$$
\begin{gathered}
L=\iint_{Q^{\prime}(0,1)} d y \frac{d t}{t} \\
\theta(x)=L^{-1} \iint_{Q^{\prime}(0,1)}(\varphi)_{t}(y-x) d y \frac{d t}{t}
\end{gathered}
$$

and

$$
k(y, t)=(\check{\varphi}-\theta) *(\check{\psi})_{t}(y)+L^{-1} \chi_{Q^{\prime}(0,1)}(y, t) .
$$

Since

$$
\int \check{\varphi}(x)-\theta(x) d x=0
$$

and since $c(\check{\varphi}-\theta) \in \mathscr{B}_{\alpha}^{\prime}\left(R^{n}\right)$ with $c>0$ small enough, Remark 5.2 implies that $c(\check{\varphi}-\theta) \in \mathscr{B}_{\alpha}^{0 \prime}\left(R^{n}\right)$ if $c>0$ is small enough. Applying Lemma 5.5 (with $\nu=\alpha$ ) gives

$$
\left|(\check{\varphi}-\theta) *(\check{\psi})_{t}(y)\right| \leq C k_{\alpha, \varepsilon^{\prime}}(y, t) .
$$

So,

$$
|k(y, t)| \leq C k_{\alpha, \varepsilon^{\prime}}(y, t) .
$$

By $(5.6)^{\prime}$ and by the definition of $\theta$ we get

$$
\begin{aligned}
\iint_{R_{+}^{n+1}}(\varphi)_{t}(y-x) k(y, t) d y \frac{d t}{t} \\
=(\check{\varphi}(x)-\theta(x))+\theta(x)=\varphi(-x) .
\end{aligned}
$$

Similarly put

$$
\eta(x)=\mathscr{F} \kappa(0) \mathscr{F} \varphi(0)^{-1} L^{-1} \iint_{Q^{\prime}(0,1)}(\varphi)_{t}(y-x) d y \frac{d t}{t}
$$

and

$$
k^{1}(y, t)=(\kappa-\eta) *(\check{\psi})_{t}(y)+\mathscr{F} \kappa(0) \mathscr{F} \varphi(0)^{-1} L^{-1} \chi_{Q^{\prime}(0,1)}(y, t) .
$$

Since

$$
\int \kappa(x)-\eta(x) d x=0
$$


and since $c(\kappa-\eta) \in \mathscr{B}_{\beta}^{\prime}\left(R^{n}\right)$ with $c>0$ small enough (recall Remark 1.1), Remark 5.2 implies that $c(\kappa-\eta) \in \mathscr{B}_{\beta}^{0 \prime}\left(R^{n}\right)$ if $c>0$ is small enough. Applying Lemma 5.5 (with $\nu=\beta$ ) gives

$$
\left|(\kappa-\eta) *(\check{\psi})_{t}(y)\right| \leq C k_{\beta, \varepsilon}(y, t) \text {. }
$$

Thus

$$
\left|k^{1}(y, t)\right| \leq C k_{\beta, \varepsilon}(y, t) .
$$

By $(5.6)^{\prime}$ and by the definition of $\eta$ we get

$$
\begin{aligned}
\iint_{R_{+}^{n+1}}(\varphi)_{t}(y-x) k^{1}(y, t) d y \frac{d t}{t} & \\
= & (\kappa(x)-\eta(x))+\eta(x)=\kappa(x) .
\end{aligned}
$$

(6.3) and (6.4) correspond to (5.23) and (5.24). (6.5) and (6.6) correspond to (5.25) and (5.26) of the case $m=1$. Then, the rest is the same with the preceding section.

Inductively we define

$$
k^{m}(y, t)=\iint_{\mathscr{E}} k^{m-1}(x, s) s^{-n} k\left(\frac{y-x}{s}, \frac{t}{s}\right) d x \frac{d s}{s}
$$

for $m=2,3,4, \ldots$, which corresponds to (5.22). Then by the same argument with the preceding section we get

$$
\left|k^{m}(y, t)\right| \leq C(C a)^{m-1} k_{\beta, \varepsilon}(y, t)
$$

and

$$
\begin{aligned}
\kappa(x)= & \iint_{\mathscr{E}^{c}}(\varphi)_{t}(y-x) \sum_{l=1}^{m} k^{l}(y, t) d y \frac{d t}{t} \\
& +\iint_{\mathscr{E}}(\varphi)_{t}(y-x) k^{m}(y, t) d y \frac{d t}{t}
\end{aligned}
$$

which correspond to (5.25)-(5.26). Put

$$
k(y, t)=\sum_{m=1}^{\infty} k^{m}(y, t) .
$$

If $C_{6.1}$ is small enough, then by (6.7) this converges everywhere and satisfies (6.1). Letting $m \rightarrow \infty$ in (6.8) gives (6.2).

\section{REFERENCES}

[1] A. Calderón and A. Torchinsky, Parabolic maximal functions associated with a distribution, Advances in Math., 16 (1975), 1-63.

[2] R. Coifman and G. Weiss, Extensions of Hardy spaces and their use in analysis, Bull. Amer. Math. Soc., 83 (1977), 569-646. 
[3] P. Duren, Extension of a theorem of Carleson, Bull. Amer. Math. Soc., 75 (1969), 143-146.

[4] C. Fefferman, N. Riviere and Y. Sagher, Interpolation between $H^{p}$ spaces, the real method, Trans. Amer. Math. Soc., 191 (1974), 75-81.

[5] C. Fefferman and E. M. Stein, Some maximal inequalities, Amer. J. Math., 93 (1971), 107-115.

[6] _ $H^{p}$ spaces of several variables, Acta Math., 129 (1972), 137-193.

[7] R. Fefferman and E. M. Stein, Singular integrals on product spaces, Advances in Math., 45 (1982), 117-143.

[8] G. H. Hardy and L. E. Littlewood, Some properties of conjugate functions, J. für Mathematick, 167 (1932), 405-423.

[9] E. M. Stein, Singular Integrals and Differentiability Properties of Functions, Princeton, 1970.

[10] M. Taibleson and G. Weiss, The molecular characterization of certain Hardy spaces, Asterisque, 77 (1980), 67-149.

[11] A. Torchinsky, On a mean value inequality, Bull. Amer. Math. Soc., 81 (1975), 950-953.

[12] A. Uchiyama, A maximal function characterization of $H^{p}$ on the space of homogeneous type, Trans. Amer. Math. Soc., 262 (1980), 579-592.

[13] _ Grand maximal functions and radial maximal functions, preprint.

[14] $\_$, Characterization of $H^{p}\left(R^{n}\right)$ in terms of generalized Littlewood-Paley g-functions, to appear in Studia Math.

Received January 4, 1984. Supported in part by NSF MCS-8203319 USA and Grant-in-Aid for Scientific Research No. 59740056 JAPAN.

TOHOKU UNIVERSITY

SENDAI, 980, JAPAN 



\section{PACIFIC JOURNAL OF MATHEMATICS EDITORS}

V. S. VARAdarajan (Managing Editor)
University of California
Los Angeles, CA 90024
CHARLES R. DePrima
California Institute of Technology
Pasadena, CA 91125
R. FINN
Stanford University
Stanford, CA 94305

V. S. VARADARAJAN (Managing Editor)

Hermann FlaschKa

University of Arizona

Tucson, AZ 85721

RAMESH A. GANGOlli

University of Washington

Seattle, WA 98195

ROBION KIRBY

University of California

Berkeley, CA 94720
C. C. MOORE

University of California

Berkeley, CA 94720

H. SAMELSON

Stanford University

Stanford, CA 94305

Harold Stark

University of California, San Diego

La Jolla, CA 92093

\section{ASSOCIATE EDITORS}
R. ARENS
E. F. BECKENBACH
B. H. NeumanN
F. WOLF
K. Yoshida (1906-1982)

\section{SUPPORTING INSTITUTIONS}

\author{
UNIVERSITY OF ARIZONA \\ UNIVERSITY OF BRITISH COLUMBIA \\ CALIFORNIA INSTITUTE OF TECHNOLOGY \\ UNIVERSITY OF CALIFORNIA \\ MONTANA STATE UNIVERSITY \\ UNIVERSITY OF NEVADA, RENO \\ NEW MEXICO STATE UNIVERSITY \\ OREGON STATE UNIVERSITY
}

UNIVERSITY OF OREGON UNIVERSITY OF SOUTHERN CALIFORNIA

STANFORD UNIVERSITY

UNIVERSITY OF HAWAII

UNIVERSITY OF TOKYO

UNIVERSITY OF UTAH

WASHINGTON STATE UNIVERSITY

UNIVERSITY OF WASHINGTON 


\section{Pacific Journal of Mathematics}

\section{Vol. 120, No. $1 \quad$ September, 1985}

Ulrich F. Albrecht, A note on locally $A$-projective groups $\ldots \ldots \ldots \ldots \ldots 1$

Marilyn Breen, A Krasnosel'skiü-type theorem for unions of two starshaped

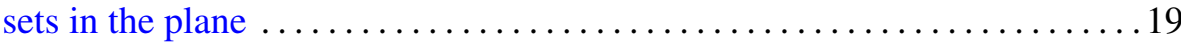

Anthony Carbery, Sun-Yung Alice Chang and John Brady Garnett,

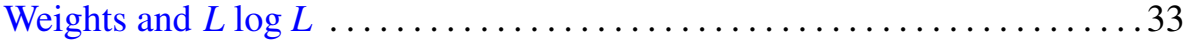

Joanne Marie Dombrowski, Tridiagonal matrix representations of cyclic

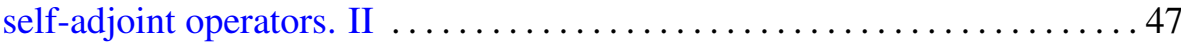

Heinz W. Engl and Werner Römisch, Approximate solutions of nonlinear random operator equations: convergence in distribution $\ldots \ldots \ldots \ldots \ldots 55$

P. Ghez, R. Lima and J. E. Roberts, $W^{*}$-categories $\ldots \ldots \ldots \ldots \ldots \ldots$

Barry E. Johnson, Continuity of homomorphisms of Banach $G$-modules . . 111 Elyahu Katz and Sidney Allen Morris, Free products of topological groups

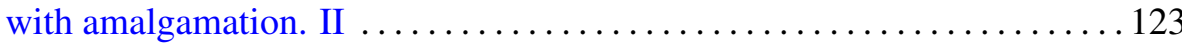

Neal I. Koblitz, $p$-adic integral transforms on compact subgroups of $\mathbf{C}_{p} \ldots 131$ Albert Edward Livingston, A coefficient inequality for functions of positive real part with an application to multivalent functions $\ldots \ldots \ldots 139$

Scott Carroll Metcalf, Finding a boundary for a Hilbert cube manifold

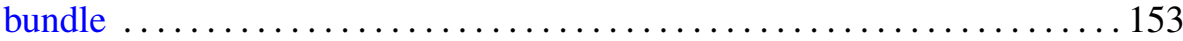

Jack Ray Porter and R. Grant Woods, When all semiregular $H$-closed extensions are compact ........................

Francisco José Ruiz and José Luis Torrea, A unified approach to Carleson measures and $A_{p}$ weights. II $\ldots \ldots \ldots \ldots \ldots \ldots \ldots \ldots \ldots \ldots \ldots \ldots \ldots$

Timothy DuWayne Sauer, The number of equations defining points in general position

John Brendan Sullivan, Universal observability and codimension one subgroups of Borel subgroups

Akihito Uchiyama, Extension of the Hardy-Littlewood-Fefferman-Stein inequality 